\title{
Bio-inspired control of an arm exoskeleton joint with active-compliant actuation system
}

\author{
Michele Folgheraiter $^{\mathrm{a} *}$, Jose de Gea ${ }^{\mathrm{b}}$, Bertold Bongardt ${ }^{\mathrm{a}}$, Jan Albiez ${ }^{\mathrm{a}}$ and Frank Kirchner ${ }^{\mathrm{a}, \mathrm{b}}$ \\ ${ }^{a}$ DFKI (German Research Center for Artificial Intelligence) Bremen, Germany; ${ }^{b}$ University of Bremen, Robotics Group \\ Robert-Hooke-Strasse, Bremen, Germany
}

(Received 30 September 2008; final version received 10 March 2009)

\begin{abstract}
This paper presents the methodology followed on the design of a multi-contact point haptic interface that uses a bio-inspired control approach and a novel actuation system. The combination of these components aims at creating a system that increases the operability of the target, and, at the same time, enables an intuitive and safe tele-operation of any complex robotic system of any given morphology. The novelty lies on the combination of a thoughtful kinematic structure driven by an active-compliant actuation system and a bio-inspired paradigm for its regulation. Due to the proposed actuation approach, the final system will achieve the condition of wearable system. On that final solution, each joint will be able to change its stiffness depending on the task to be executed, and on the anatomical features of each individual. Moreover, the system provides a variety of safety mechanisms at different levels to prevent causing any harm to the operator. In future, the system should allow the complete virtual immersion of the user within the working scenario.
\end{abstract}

Keywords: exoskeleton; haptic interface; biomimetic robotics; compliant joint; stiffness controller; neural controller

\section{Introduction}

In recent years, a growing interest has been observed on the use of artificial exoskeletons, especially on medical applications and where hazardous industrial environments are found. Although the use of artificial exoskeletons was common in the Medieval times, where knights would use them to protect themselves in combat, the construction of an active and really wearable exoskeleton still, nowadays, presents several challenges.

Artificial exoskeletons are deployed, inter alia, in virtual reality scenarios (Frisoli et al. 2005; Perry and Rosen 2006) teleoperation environments (Caldwell et al. 1995; Kim et al. 2001; Schiele and Visentin 2003), and for medical therapy and diagnosis (Carignan et al. 2005; Gupta and O'Malley 2006; Nef et al. 2005; Riener et al. 2005; Tsagarakis and Caldwell 2003). To translate the forces occurring in the virtual world or the teleoperation environment onto the carrier of the exoskeleton, they must monitor the movements of the carrier and every joint must be fitted with actuators, which can exercise force, yet at the same time be light and compact (i.e. possessing a large power to weight/volume ratio) to be able to perform force feedback on the carrier. The actuators must, furthermore, be fast enough to achieve an appropriate force/rigidity, but may not, in any way, create forces that endanger the carrier. Brown et al. (2006) provide an overview of the current research on exoskeletons for force augmentation. Similarly, Pons (2008) provides a thorough overview on exoskeleton technologies, with a fo- cus on biologically inspired approaches for their design and control.

The choice of the type of actuator is a conflict of goals between the above-mentioned demands. At the same time, current state-of-the-art actuators fail at providing some desired properties as being strong, compact and with the capability to actively change its impedance (Alleyne 1996). Hydraulic actuators provide a number of advantages over electrical motors, such as higher force-to-weight ratio without the need for gear boxes, faster response time, accurate position control (the fluid is not compressible) and a compact size. The main problem of these systems is, however, their high non-linearity and, as a result, a more complex control system. Also when the device is not operating it presents a high stiffness that may be a problem for the safety of the user. For this reason, a series of actuation techniques has been tested for exoskeletons. These are, among others, pneumatic (Lee et al. 1998; Tsagarakis et al. 1999), hydraulic and piezoelectric actuators, as well as shape memory alloy, magnetic and electronic brakes (Kim et al. 2001). Electric motors are favoured for most of the current systems, as they are the simplest to integrate and control. On the other hand, electric motors are characterised by a low power-to-weight ratio so that they usually have to be connected with drive-gears, which creates additional disadvantages, such as backlash and friction. To compensate for this, harmonic drive gears without backlash are used, which, however, leads to heavy and cumbersome systems.

\footnotetext{
*Corresponding author. Email: Michele.folgheraiter@dfki.de
} 
In such comparatively heavy systems, the motor controller has to compensate for gravity so that the system appears lighter to the operator. Another alternative to achieve light designs is to place the actuators on the base and drive the joints using cables, as in (Schiele and Veneman et al. 2006).

This work presents a new design based upon the combination of three aspects: human kinematic analysis, activecompliant actuator based on a hydraulic plus pneumatic actuator and design of a control system based on biological principles. The idea of providing with compliance to the highly stiff hydraulic actuators has been already researched in several works using different approaches. For instance, McKinney pneumatic actuators are used in parallel with an hydraulic damper (Klute and Czerniecki 1999), seeking a muscle-like behaviour of an artificial actuation system. Series Elastics Actuators (SEAs) (the combination of an elastic element in series with the actuator) have been also applied to an hydraulic actuator (Robinson 2000). Similarly, an hydraulic actuator plus mechanical spring has also been used in legged robots (Raibert 1986). Although in this case, the purpose was not to directly control the stiffness, but to store and use the impact energy to produce a more efficient walking. A completely different approach is found in Alleyne (1996), where a non-linear force controller is used to control a hydraulic piston and offer compliance to the system. In the area of exoskeletons, the robot SARCOS (Jacobsen et al. 1990), the first force-reflective teleoperated robot using hydraulic actuation, was later enhanced to include compliance capabilities (Wells et al. 1990). In this case, accumulators were used inside the hydraulic pistons to provide with compliance, that is, no mechanical spring was used but the intrinsic fluid spring.

The paper is organised as follows: Section 2 deals with the kinematic model of the exoskeleton, in particular the system to be coupled with the shoulder of the user; Section 3 describes the model of the actuation system and the experimental results; Section 4 describes the bio-inspired control system for the single exoskeleton joint and finally, Section 5 presents conclusions and further areas of work. An appendix is also present for readers that would like to enter into more detail regarding the exoskeleton design.

\section{The exoskeleton kinematics and design}

As stated in the previous section, the main goal of this work is to develop a multi-contact point haptic interface for the human upper limb. Since the aim is to provide with a specific force feedback at different locations of the user's arm, a carefully designed kinematic configuration is required. Another consideration to bear in mind is to obtain a design that restricts the user's mobility minimally, and therefore, enhances his workspace. Following this direction, if the exoskeleton is made wearable, the operability of the slave device can be increased by virtually immersing the operator into the working scenario.

The design of such a device still, nowadays, poses great challenges due to a number of reasons. First, in order not to restrict the arm movement, the kinematic complexity of the exoskeleton increases to a level that is comparable to the complexity of the human arm itself. Second, delivering a complex force pattern on the human arm requires the possibility to actuate different joints of the exoskeleton. Those conditions lead to a complex architecture for the actuation system, and obviously to a heavier device.

As an example for the kinematic design of the exoskeleton, this section introduces the approach followed on the design of the shoulder joint. On the one hand, it is necessary to define the contact points where the force feedback is applied. Figure 11 depicts three of these points. One is located between the exoskeleton-shoulder and the usershoulder, the other is located on the middle of the user's upper arm and the last one in the middle of the user's forearm. These locations are optimal in the sense that they reduce the interference with the human articulation during the user movements.

On the other hand, the kinematics of the exoskeleton has to be defined which is strongly influenced by the human arm kinematics. After a study of the human shoulder anatomy and osteology, a kinematic model is extracted and formalised using robotic notation. This allows the test of different possible kinematic chains for the exoskeleton. The chosen configuration is presented in Figure 1, showing a complex structure with different closed kinematic paths.

In order to study this system, a first simplification is made by substituting the upper shoulder kinematic with a single three degree of freedom (DoF) joints. To calculate the mechanism mobility $M$, the Chebychev-GrueblerKutzbach formula for spatial configurations (Gogu 2005) is used.

$$
M=\sum_{i=1}^{p} f_{i}-b \cdot q
$$

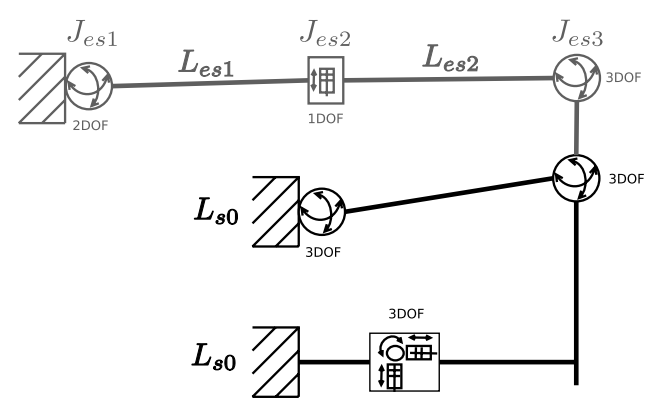

Figure 1. Closed kinematic chain formed between the exoskeleton shoulder joint and the upper shoulder kinematic chain. Notation convention: $J$ stands for 'joint', $L$ for 'link', $s$ for 'shoulder' and $e$ for 'exoskeleton'. 
Thereby, $f_{i}$ is the degree of freedom of the $i$ th joint ( $i=1, \ldots, p), b=6$ is the 'mobility number of spatial mechanisms' (Gogu 2005) and $q$ is the number of independent loops, where $q=1$ in this case. For this simplified configuration, the mobility results in:

$$
M=(2+1+3+1+2)-6 \cdot 1=9-6=3 .
$$

As is represented in Figure 1, the exoskeleton has a total of six DoF; since only a three-DoF kinematic chain needs to be actuated, only three of the six DoF must be actuated and sensed. From a mechanical point of view, it is more logical to actuate the joints closer to the body's barycenter, that is, $J_{e s 1}$ and $J_{e s 2}$. By doing that, the actuation system is not required to move the weight of the actuators (notice the extra torque that would be required on joint $J_{e s 1}$, caused by a weight located near the joint $J_{e s 3}$ ).

A first passive version of the device was developed in order to test on a real subject of the chosen kinematic configuration. The system has the same mobility of the model, and each joint is equipped with an angular position sensor. More information is provided in Appedix 5

\section{Hydraulic actuation system with pneumatic-compliant elements}

As previously stated, one of the main purposes is to implement and test a hybrid actuation system that is suitable for the haptic interface being developed. In the proposed concept (Figure 2), a 'pneumatic spring' is connected in series with the hydraulic actuator. Due to this active elastic element, the stiffness of the joint can be regulated without disturbing its position. When more precision is required in regulating the joint position, it is possible to change the characteristic of the pneumatic spring via its inner pressure. The increase of the pressure also augments the initial force required to start the compression of the spring. This turns out in having a spring that behaves more like a rigid link; in other word, during a normal movement, the forces in play will not be able to significantly change the spring po- sition any more. A local controller measures the positions and pressures of the hydraulic and pneumatic elements and controls, via the electrovalves, the position and stiffness of the joint.

In order to study this complex system, the different components were individually modelled, and some of these models were later validated via experimentation. In those experiments, the hydraulic part of the system was kept passive, that is, no force was generated by the hydraulic actuator. Nevertheless, the fluid flow between the two piston chambers was regulated, and therefore, it was possible to examine the damping behaviour of the system.

The test bed built to explore the different components is shown in Figure 3. Both the data acquisition and the control strategy were implemented using the real-time Windows Toolbox of Matlab. The sample frequency was set to $1 \mathrm{kHz}$. The experimental set-up is composed of the following elements: A hydraulic rotary actuator, a hydraulic valve, a lever to apply force on the actuator, an absolute position sensor, a relative encoder, two pneumatic solenoid valves, a force sensor and two hydraulic and pneumatic pressure sensors. An external force is required in order to move the cylinder's rod.

The following section will describe the pneumatic spring implementation, whereas its bio-inspired control system is discussed later in Section 4.

\subsection{The pneumatic spring}

The possibility to have a physical elastic element in series with the actuator revealed to be very important, especially in applications where it is necessary to keep the system with a low stiffness, or where it is required to absorb the impact energy due to an unexpected load (Veneman et al. 2006). In the particular case of an exoskeleton, it may also happen that due to the failure of some component of the system (sensor, control module, power etc.), the human limb is forced rapidly to postures that are not natural, thus being also dangerous. If that occurs, an intrinsic passive mechanism is required that prevents the system from injuring the

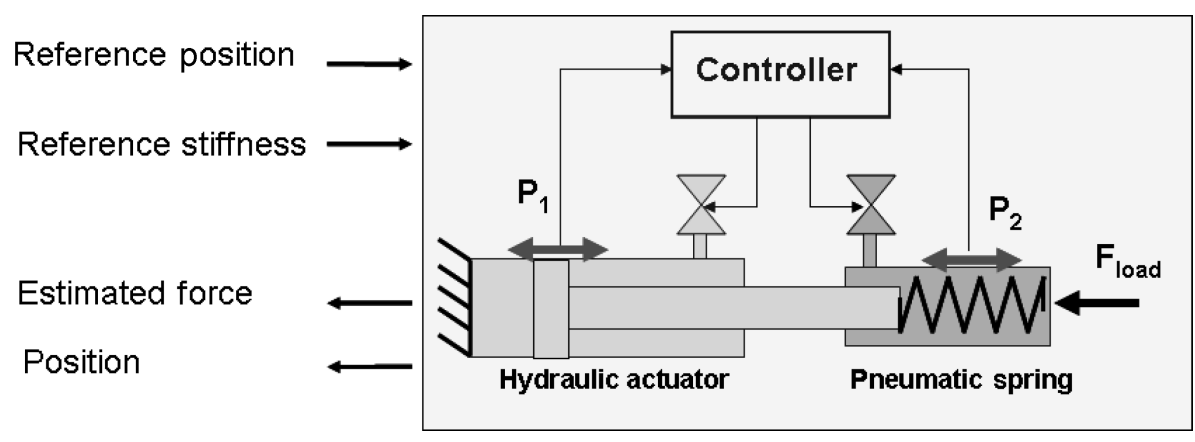

Figure 2. A concept schema for the hybrid hydraulic-pneumatic actuation system. 

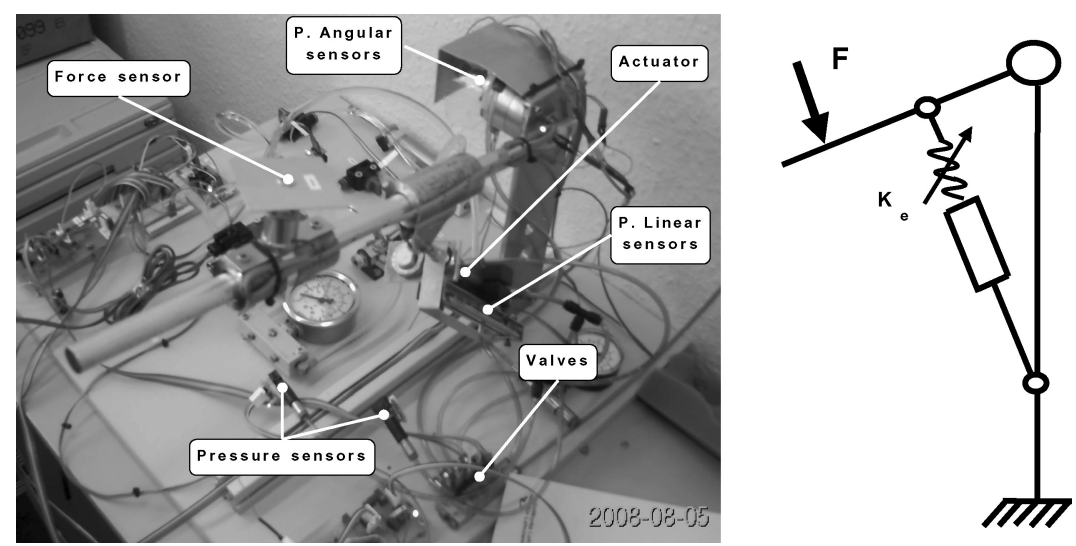

Figure 3. The test bed built to explore the actuator concept (left side). A simplified schema of the hydraulic actuator connected in series with the pneumatic spring, the user can apply a force according to the convention in the schema (right side).

user. Having a spring in series with the actuator provides this functionality. For example, if a spring of stiffness $K$ and maximum contraction $\Delta y$ is included in series with the actuator, and the actuator has a excursion $E \leq \Delta y$, the maximum force delivered to the load is $F_{L} \leq K \cdot \Delta y$. Despite the advantages provided by this solution, the control strategy needs to take into account this elastic element, and thus the control system will be more complex.

The main drawback of applying a mechanical spring is the impossibility of actively controlling its elastic constant. Given the scenario when stiffness modulation is required, the control system needs to apply a specific strategy to the actuator-spring system to recreate a stiffness for the load. In this particular case, the stiffness is controllable only if a load is applied to the system (Wells et al, 1990). Moreover, if the position needs to be controlled at the same time, a compromise has to be found. The capability to change the stiffness of the exoskeleton joint actively is also required due to the fact that different users of the system have different muscular structure. This turns into the requirement that the maximum deliverable force should change depending on the user's features. The proposed design aims at exploiting the elasticity properties of the air, thus designing a physical element with variable and controllable stiffness.

In order to understand this possibility better, a simplified model of the pneumatic spring element is considered. The isolated system represented in Figure 4 allows us to

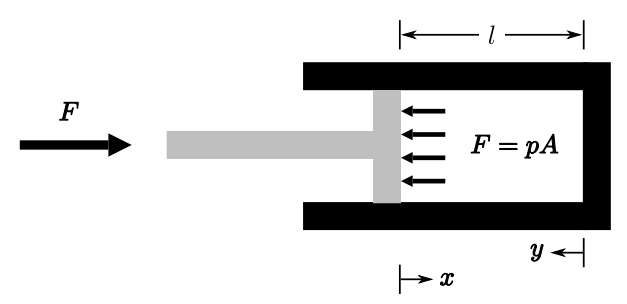

Figure 4. The pneumatic chamber with variable volume. calculate the forces required to compress the ideal gas to a certain volume $V$.

The static model of this system can be formulated combining the equation of the ideal gas (Equation 3) and the equations that relate pressure, volume and force inside the pneumatic chamber (Equation 4).

$$
\begin{aligned}
p V & =n R T . \\
F & =p A \\
V & =A y .
\end{aligned}
$$

After some simplifications that consider the initial volume and pressure, one obtains the anti-linear relationship between the length $y$ and the force $F$. Equation 5 reads that the smaller the distance $y$ becomes, the bigger force $F$ grows. The elastic constant $K$ is computed considering air at a constant temperature of $293 \mathrm{~K}$, initial pressure of $10^{5} \mathrm{~Pa}$ and the universal gas constant $R=8.3 \mathrm{~J}(\mathrm{~mol} \mathrm{~K})^{-1}$.

$$
F=\frac{n R T}{y}=\frac{K}{y} .
$$

It is also possible to apply a substitution of variables to bring Equation 5 to the conventional form of the spring formula $F=k x$. By observing Figure 4, it follows that $y=l-x$, and therefore, $F=\frac{K}{l-x}$. Figure 5 depicts this relationship for different pressures. The cylinder is assumed to have a diameter of $0.01 \mathrm{~m}$ and a stroke $l$ of $0.07 \mathrm{~m}$.

Note that if the movement is limited to the first part of the characteristic (until the $30 \%$ of the maximum compression), the behaviour can be approximated as linear. In order to test the feasibility of this system, a first experiment was conducted where the force generated by the pneumatic spring is measured at different lengths. A light pneumatic cylinder is employed that has an internal diameter of $1.10^{-2} \mathrm{~m}$ and a stroke of $0.1 \mathrm{~m}$. Position and force were 

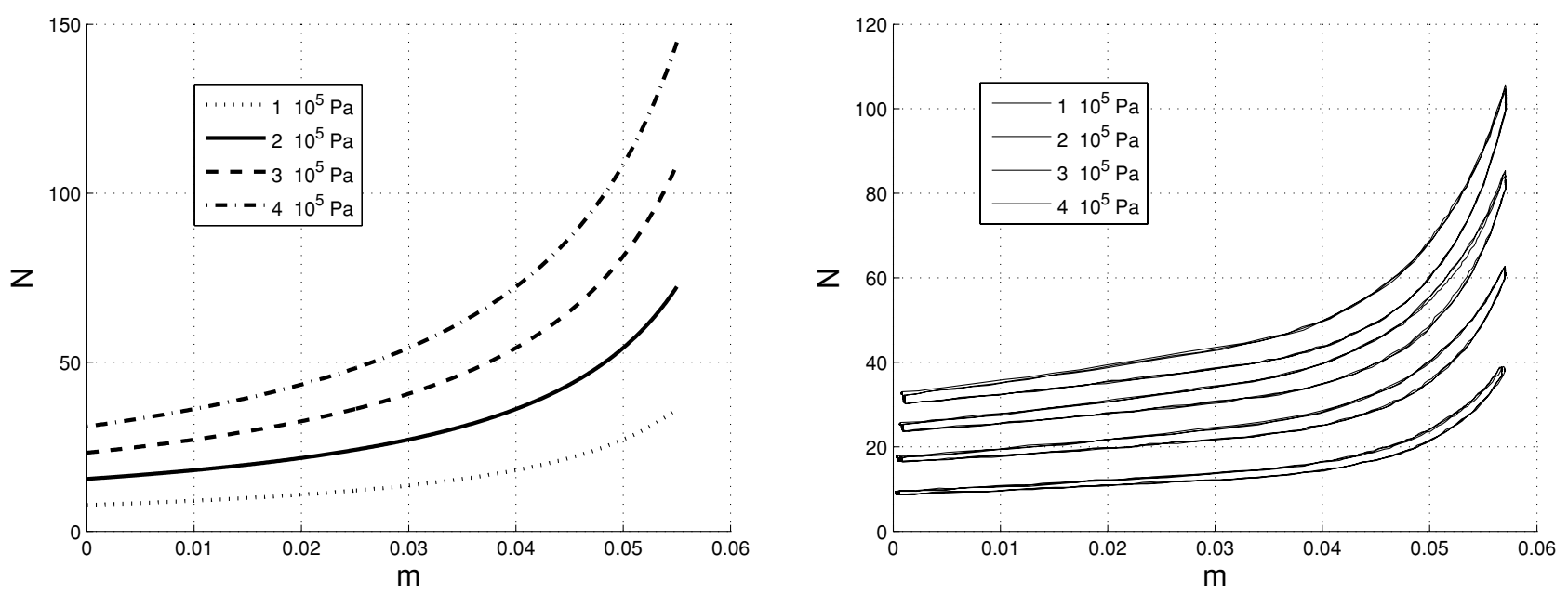

Figure 5. Relationship between force and length change in a pneumatic spring. Left: simulation results, right: experimental results.

acquired with a sampling time of $1 \mathrm{~ms}$. Figure 5 shows the results for four pressures $(100,200,300$ and $400 \mathrm{kPa})$. As it can be seen, the force trend is quite similar to the formulated model, with only a small discrepancy on the values that might originate from the fact that friction and damping effects have not been included in the model. As it can be seen from Figure 5, a hysteresis phenomenon is also evident, probably due to the presence of a hysteresis in the tube.

\subsection{The hydraulic actuator}

A second experiment was conducted with the intent to start analyse the behaviour of the hydraulic components, and to evaluate a first position control loop. At this time different crucial components of the hydraulic circuit were operative.

The hydraulic pump. This component is required in order to generate pressure inside the hydraulic circuit. For safety reason at the moment the pump pressure is limited at $10 \mathrm{bar}\left(10 \times 10^{5} \mathrm{~Pa}\right)$; therefore the maximum torque developed by the hydraulic actuator is limited.

The hydraulic valve. This component is intended to regulate the hydraulic flow inside the actuator in order to regulate direction, velocity and torque. The used device is a commercial three-position miniaturised solenoid valve (from Numatics ${ }^{\circledR}$, its weight is only $0.1 \mathrm{~kg}$ ) developed for pneumatic applications. In order to use it as a hydraulic valve we improve the sealing mechanics. The main advantage of this valve is the fast switching frequency, which can possibly reach up to $20 \mathrm{~Hz}$.

The rotative actuator. This component converts the hydraulic energy into a kinetic energy. The actuator generates a torque that is proportional to the pressure inside the two chambers and can rotate up to $270^{\circ}$.
The position sensor. This component measures the angular position of the actuator.

A proper control logic was used to switch on and off the two solenoids that equip the valve, this according to the sign of the position error. In order to regulate the position, a PID controller was added between the error calculation module and the control logic module. The three parameters for the PID controller were adjusted using the ZiglerNichols method $(P=10, I=1, D=0.06)$. The inputs for the control system were four different reference positions: $10^{\circ}, 20^{\circ}, 30^{\circ}, 45^{\circ}$. In the graph of Figure 6 it is possible to see the performance of the controller in following the references.

In some positions a steady position error also appears. This is mainly due to the fact that the PID parameters are still not optimised. It is also possible to note a noise inside the position measurement, these are some artifacts that have to be suppressed, maybe using a filter connected on the power line of the pump. In the graph of Figure 6 the pressure trend during the movements of the actuator is also represented. As it is normal to expect from a hydraulic circuit, when the position is changing the pressure inside the circuit is decreasing. In our system we have a $\Delta P$ of 2 bar $\left(2 \times 10^{5} \mathrm{~Pa}\right)$. It still remains to experiment with the combination of the hydraulic circuit and the pneumatic spring; in particular it will be very interesting to understand how the elastic element affects the precision of the control system in regulating the joint position. It is likely that the performances will be reduced as soon as the stiffness of the joint is decreased.

\section{The single joint bio-inspired control system}

The control system is responsible for actively controlling the configuration and stiffness of the exoskeleton arm when 


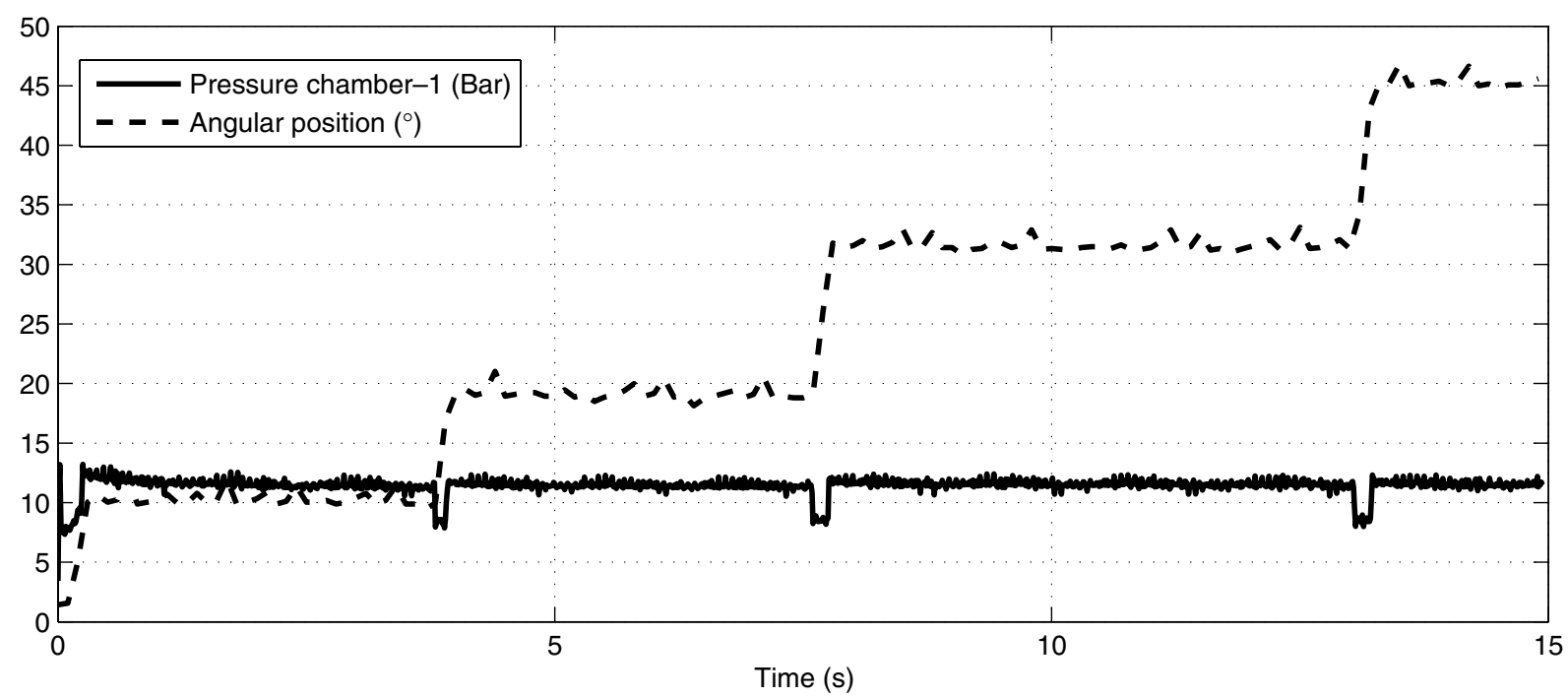

Figure 6. Angular position of the actuator and pressure trend of one of the actuator's chamber.

interacting with the user. If for some reason the robot arm performs an unexpected movement, a safety mechanism is required that avoids exerting dangerous forces to the user's arm. This safety mechanism should work any time despite any power or software failure. To our understanding, the best control strategy for a device that is coupled with the human arm should come from looking for inspiration from biological control strategies. One of the most important features to include is a mechanism that controls the maximum force that can be applied to the user. A possible solution is to include a controller on each joint which regulates the variable joint stiffness that is provided with the pneumatic actuator. From the point of view of the controller, the system is inherently not linear due to several reasons.

- The presence of a non-linear pneumatic spring in the actuation system.

- The high non-linearity of the hydraulic system itself.

- The interaction with a stochastic system, the human body.

Such a highly complex and non-linear system poses many challenges to a classical controller and, for that reason, this work also explores other control paradigms (Wells et al. 1990). Possible solutions come from the analysis of physiological studies of the human peripheral nervous system and, in particular, sensory-motor coordination strategies (Cisek et al. 1998). The stiffness of the musculoskeletal system can be finely controlled using the co-contraction muscle mechanism due to the regulation activity performed by the $\gamma$-motoneurons. Furthermore, there is also a protection mechanism that monitors the activity of the Golgi-
Tendon organs (located in series with the muscle) that measure the force the muscle applies on the bone. When an unexpectedly dangerous external load is acting on the muscle, this mechanism acts rapidly in order to decrease the muscle stiffness, thus letting the articulation move under the load. This action serves to prevent permanent damage to the muscles or the tendon tissues.

The control schema in Figure 8 represents a first trial to mimic this control architecture, here combining three different kind of control strategies: position, force, and stiffness control. These different control loops, due to the intrinsic parallel nature of the artificial neural network, can superpose and coordinate in order to perform the best action.

The position loop starts from the measurement of the total position of the linear joint. The total position is calculated by adding the position of the hydraulic actuator and that of the pneumatic spring. The error signal due to the difference of the reference position and the real position is computed and employed to excite the $\alpha$ motoneurons that govern the pressure and, therefore, the position of the hydraulic actuator. A mechanism of unbalanced autoinhibition is present between the two $\alpha$ motoneurons to coordinate their activities; when one increases its activities the other decreases its potential.

The force loop starts from the measurement of the pneumatic spring position. From this data the force applied to the load is estimated. The force signal excites the $\gamma$-motoneuron which affects the position of the hydraulic actuator via the $\alpha$ motoneurons. This loop is intended to regulate the delivered force to the load; if this force is changing, a fast feedback will alter the position of the hydraulic actuator. This action has the main effect of restoring the equilibrium position of the pneumatic 

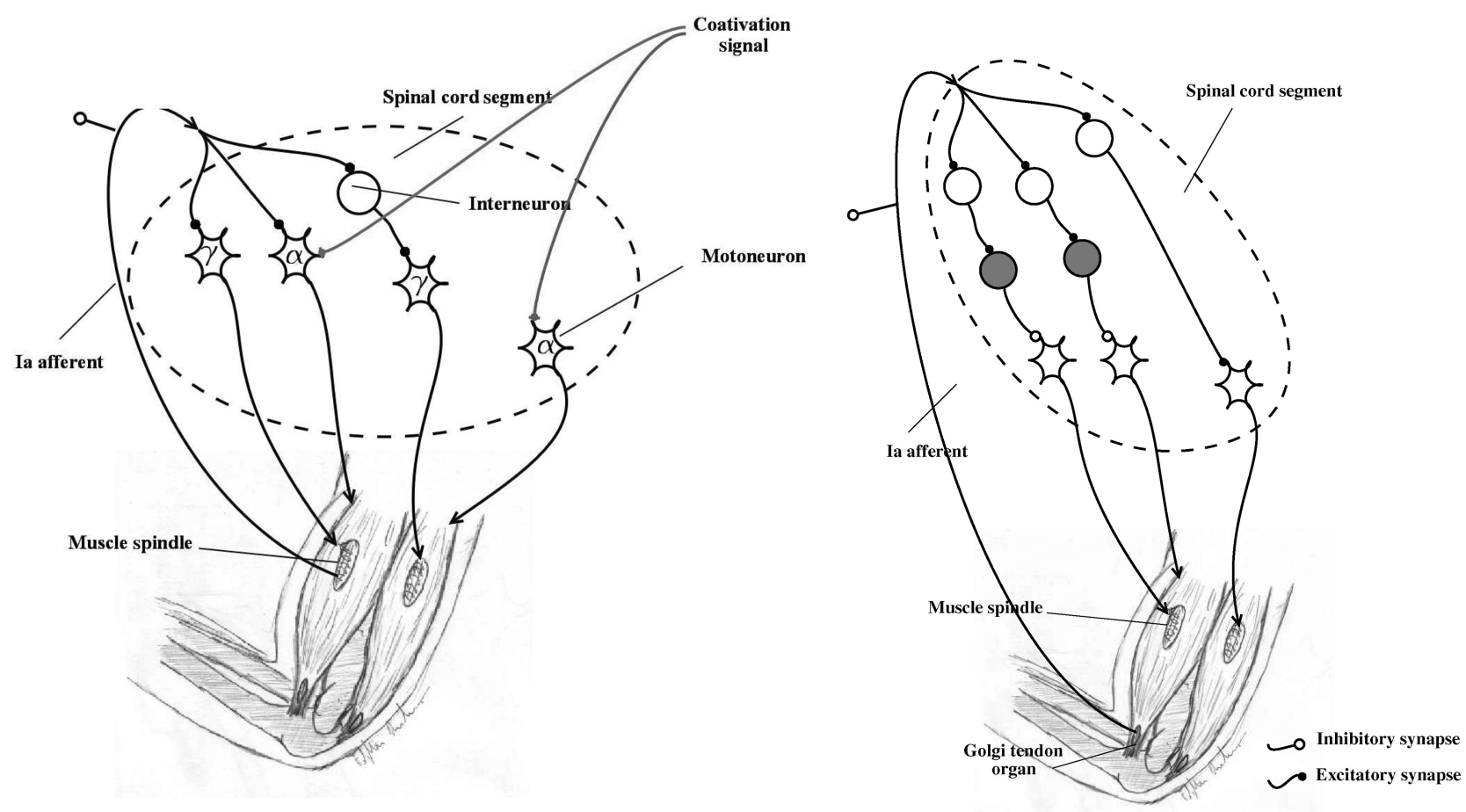

Figure 7. Two control schemes that affect the position, stiffness and maximum force for the elbow joint.

spring. A change of the force will also alter the neural circuit that regulates the stiffness of the pneumatic spring (see Figure 8).

This mechanism has some analogies with the circuit found in the natural limb, however here the architecture was redesigned in order to comply with the existing actuation system to control. In the biological $\alpha-\gamma$ loop (see Figure 7), the $\gamma$-motoneuron sets an equilibrium position for the muscle-spindle. Furthermore, the external fibers of the muscle-spindle are connected with the fibres of the muscle. That means if a load changes, the muscle length will also affect the length of the spindle. Consequently, the spindle will send a command to the $\alpha$ motoneuron that will change the contraction of the muscle, thus restoring the equilibrium position. In our architecture, the function of the spindle is replaced by a position sensor that measures the length of the pneumatic spring, therefore we have a serial configuration instead of a parallel one. Its function can also be related to the Golgi tendon organ that in the human muscle is located in the terminal part of the muscle, and measures the force that the muscle applies via the tendon to the bone. The artificial synapse that brings the position error from the pneumatic spring element to the $\gamma$-motoneuron can be modulated. That means, it is possible to modulate the effect of this error on the $\gamma$-motoneuron activity, and therefore the effect on the activity of the neural circuit responsible for controlling the overall actuator position. The model for the potential of the artificial neuron present on the circuit is reported in Equation 6.

$$
\begin{aligned}
\tau \cdot \dot{S}_{j}= & \left(A-S_{j}\right) \cdot\left(\sum \operatorname{exc}+F c\right)-\left(B+S_{j}\right) \\
& \cdot\left(\sum i n h+F c\right)
\end{aligned}
$$

where $S_{j}$ is the potential of the neuron, $\tau$ is a constant that defines the dynamics of the neuron, $F c$ is the forgetting constant, $A$ and $B$ are the maximum and the minimum values for the potential, respectively (if we chose $A=-B$ we have a bipolar neuron). $\sum e x c$ and $\sum i n h$ are the excitatory and inhibitory inputs for the neuron, respectively. The output of the neuron is shown in Equation 9, where $T h$ is a threshold function (Equation 8) that limits the output between -1 and 1 .

$$
\begin{aligned}
Y_{j} & =\operatorname{Th}\left(S_{j}\right) \\
T h(x) & = \begin{cases}x & \text { if }-1 \leq x \leq 1 \\
-1 & \text { if } x<-1 \\
1 & \text { if } x>1\end{cases}
\end{aligned}
$$

Some special neurons, called threshold neurons, are present in the circuit that regulates the stiffness of the pneumatic spring which are activated by the force signal. In this case, this neuron differs from a normal neuron on the output 


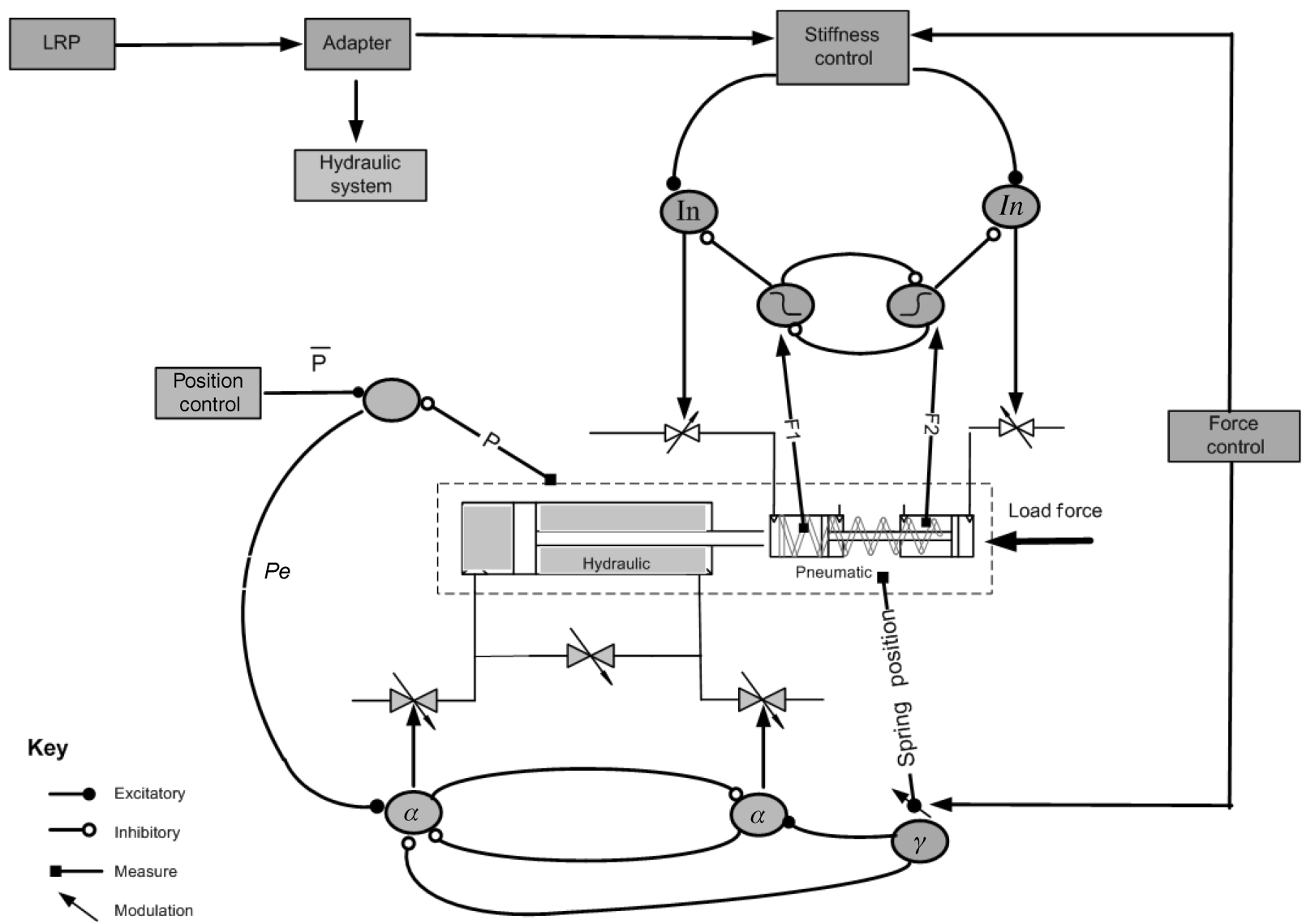

Figure 8. The bio-inspired control schema with $\alpha-\gamma$ loops.

function that is not anymore linear. The desired behaviour is a neuron that fires only when the excitatory input overcomes a certain value. An output function that can do this is the logistic function expressed in Equation 9. Modifying the parameters $a$ and $b$, the Sigmoid function can be

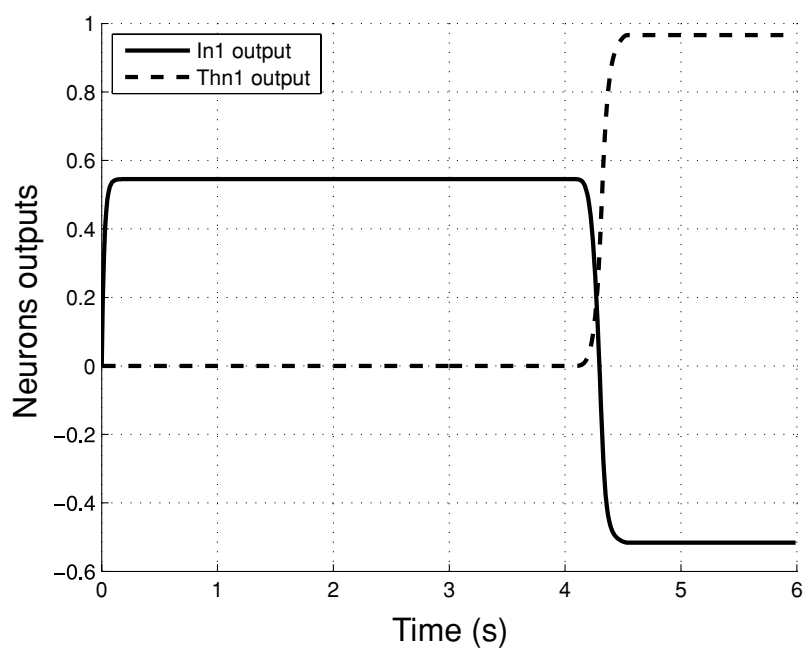

modified and set to the desired threshold.

$$
\operatorname{Sig}(x)=\frac{1}{1+e^{a-b x}}
$$

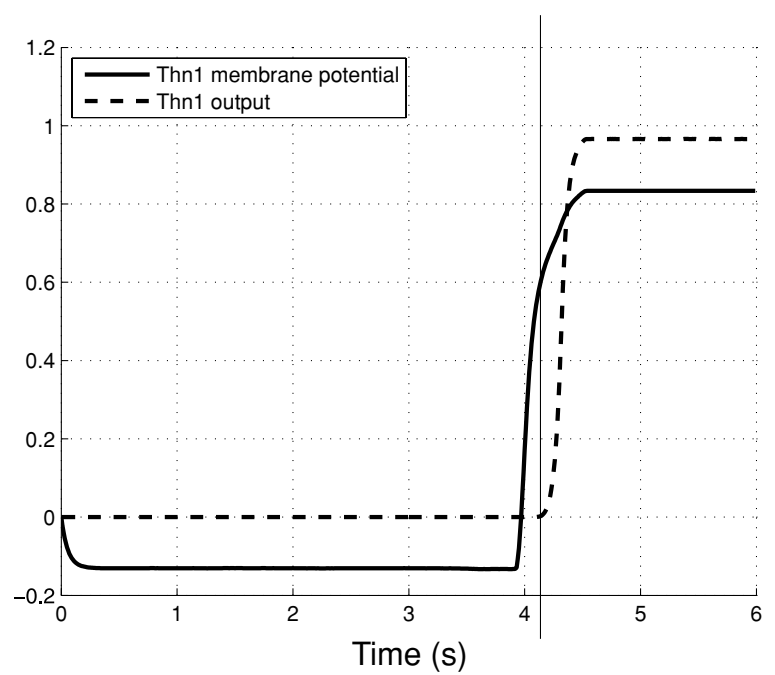

Figure 9. Activities for the neurons that regulate the pneumatic spring stiffness and implement the safe mechanism. The excitatory input that controls the stiffness was set in this experiment to 0.6. 

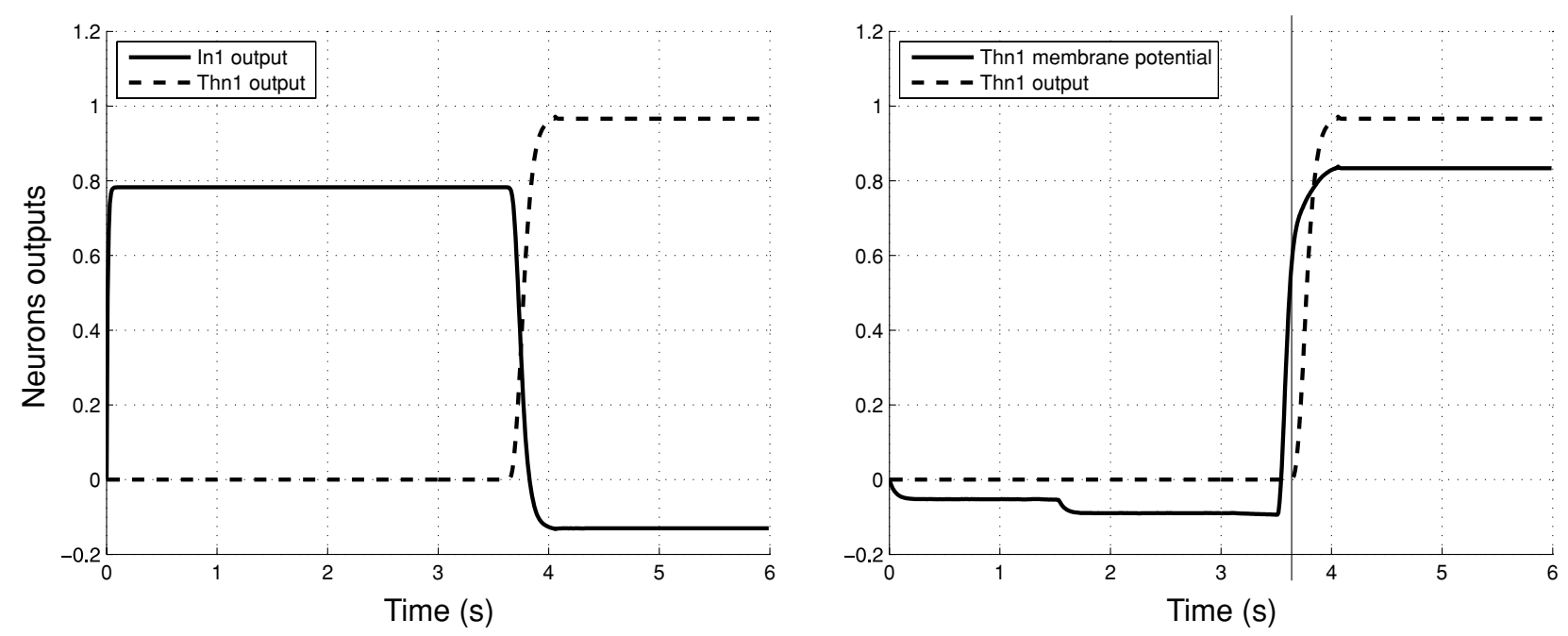

Figure 10. Activities for the neurons that regulate the pneumatic spring stiffness and implement the safe mechanism. The excitatory input that controls the stiffness was set in this experiment to 0.9 .

The third control loop of the presented architecture is the one located at the top of Figure 8. The stiffness control is possible due to two $\alpha$ neurons that control, with their activities, the pressure inside the two chambers of the spring. The presence of the two threshold neurons allows the system to modify its stiffness. When, for example, the force overcomes a certain threshold, a mechanism of auto-inhibition allows to coordinate better the two neural activities. Indeed, this cross-inhibition brings to a competitive behaviour; the neuron with the higher activity dominates the other neuron. By doing that, the spring decreases its stiffness only in the direction of the potentially dangerous force. The behaviour of this control loop was studied using the test bed shown in Figure 3. The neural network was computed in real time using a sample rate of $1 \mathrm{~ms}$, time enough to implement the dynamic behaviour of each neuron. For the model of each neuron (Equation 6), the constants were set as follows: $\tau=100, A=1, B=-1, F_{c}=0.1$ and all the input weights were set to one. For the output function of the threshold neurons, a value of $a=30$ and $b=-40$ was chosen, thus setting a threshold at $x=0.6$. The excitatory input that sets the activity of the interneuron $I_{n}$ was fixed to different values in the range 0.1 to 1 . That affects directly the activity of the neuron and, consequently, the pressure inside the pneumatic spring. A PWM function was used to convert the neural signal into proper commands to pilot the electro-valves that set the pressure inside the pneumatic spring. The pressure measurements from the pneumatic spring chambers where converted into a force, and then normalised between 0 and 1 in order to feed the excitatory inputs of the two threshold neurons.

During the experiment, the pneumatic spring was set to its maximum extension so that the control system was activated; after a few milliseconds, the neurons reached their normal activation levels. A force was applied to the pneumatic spring $4 \mathrm{~s}$ later until the safe behaviour was triggered.
The left graph of Figure 9 shows the activity of the interneuron $I_{n}$ whose output directly controls the pressure inside the chamber of the pneumatic spring. When the external force is applied, the activity of the threshold neuron increases, and since its output inhibits the interneuron $I_{n}$, this action decreases its potential and consequently the pneumatic spring stiffness. The right graph of Figure 9 depicts the potential and the output of the threshold neuron, respectively; note that when its potential overcomes the value of 0.6 , its output starts the rising very rapidly. Figure 10 shows the same experiment performed for a different value of excitatory input of the interneuron $I_{n}$; this time the threshold was set to a value of 0.9. As the right graph shows, the initial potential for the interneuron is now 0.9 , what has the effect of modifying the stiffness of the pneumatic spring.

\section{Conclusion and future work}

This paper presented the design methodology followed on the development of a multi-contact point haptic interface, as well as a novel concept for a bio-inspired actuation system. A kinematic model for the human arm shoulder is formalised and combined with the model of a possible kinematic configuration for the exoskeleton joint. The final system mobility was verified on real subjects using a passive version of the joint. The actuation system based on the combination of hydraulic actuator and active-regulated pneumatic-based spring is also presented and its stiffness regulation capabilities are shown experimentally. Moreover, a first sketch of the bio-inspired control architecture using $\alpha-\gamma$ loops is presented that mainly deals with providing safety to the user. Future work will focus on studying the kinematics of the exoskeleton system for the lower shoulder and the forearm in order to complete a first test with the whole arm. 


\section{Acknowledgements}

The work presented in this paper was done within the VI-Bot project, funded by the German Ministry of Science (BMBF), grant No. 01IW07003.

\section{References}

Alleyne A. 1996. Nonlinear force control of an electro-hydraulic actuator. Paper presented at: Japan/USA Symposium on Flexible Automation.

Brown M, Tsagarakis N, Caldwell DG. 2006. Exoskeletons for human force augmentation. Ind Robot: Intern J. 30:592602.

Caldwell D, Kocak O, Andersen U. 1995. Multi-armed dexterous manipulator operation using glove/exoskeleton control and sensory feedback. In: Proceedings of the International Conference on Intelligent Robots and Systems.

Carignan C, Liszka M, Roderick S. 2005. Design of an exoskeleton with scapula motion for shoulder rehabilitation. In: IEEE International Conference on Advanced Robotics (ICAR).

Cisek P, Grossberg S, Bullock D. 1998. A cortico-spinal model of reaching and proprioception under multiple task constraints. $J$ Cogn Neurosci, 10(4):425-444.

Contreras-Vidal J, Grossberg S, Bullock D. 1997. A neural model of cerebellar learning for arm movement control: cortico spino cerebellar dynamics. Boston (MA): Boston University. Tech. Rep. CAS/CNS-TR97/003; April.

Frisoli A, Rocchi F, Marcheschi S, Dettori A, Salsedo F, Bergamasco M. 2005. A new force-feedback arm exoskeleton for haptic interaction in virtual environments: In: WHC 05, Proceedings of the First Joint Eurohaptics Conference and Symposium on Haptic Interfaces for Virtual Environment and Teleoperator Systems, pp. 195-201.

Gogu G. 2005. Chebychev-grübler-kutzbach's criterion for mobility calculation of multi-loop mechanisms revisited via theory of linear transformations. Eur J Mech - A/Solids. 24(3):427441.

Gupta A, O'Malley M. 2006. Design of a haptic arm exoskeleton for training and rehabilitation. In IEEE/ASME Trans Mechatron. 11:280-289.

Jacobsen S, Smith C, Backman D, Iversen E. 1990. High performance, high dexterity, force reflective teleoperator. Paper presented at Conf. Remote Systems Technology.

José PL. 2005. Emerging actuator technologies, a micromechatronic approach. San Francisco(CA): John Wiley and Son, Ltd.

Kim YS, Lee S, Cho C, Kim M, Lee C-W. 2001. A new exoskeleton-type masterarm with force reflection based on the torque sensor beam. In: Proceedings of the IEEE International Conference on Robotics and Automation (ICRA).

Klute BHGK, Czerniecki JM. 1999. Mckibben artificial muscles: pneumatic actuators with biomechanical intelligence. Paper presented at: IEEE/ASME International Conference on Advanced Intelligent Mechatronics.

Lee S, Park S, Kim M, Lee C-W. 1998. Design of a force reflecting master arm and master hand using pneumatic actuator: ICRA. 2574-2579.

Nef T, Colombo G, Riener R. 2005. Armin-robot for movement therapy of the upper extremities. Automatisierungstechnik. 53(December):597-606.

Perry J, Rosen J. 2006. Design of a 7 degree-of-freedom upperlimb powered exoskeleton. In: Proceedings of the 2006 BioRob Conference.

Pons J. 2008. Wearable robots: biomechatronic exoskeletons. San Francisco (CA): John Wiley and Sons Ltd.
Raibert M. 1986. Legged robots that balance, Cambridge: MIT Press.

Riener R, Nef T, Colombo G. 2005. Robot-aided neurorehabilitation for the upper extremities. Med Biol Eng Comput. 43(Jan):2-10.

Robinson DW, Pratt JE, Paluska DJ, Pratt GA. 1999. Series elastic actuator development for a biomimetic walking robot. In: IEEE/ASME International Conference on Advanced Intelligent Mechatronics, Sep 19-22, Atlanta GA.

Robinson GPDW. 2000. Force controllable hydro-elastic actuator. Paper presented at: IEEE International Conference on Robotics and Automation.

Scarfogliero U, Folgheraiter M, Gini G. 2004. Advanced step in biped robotics: innovative design and intuitive control through spring-damper actuator. In Proceedings of IEEE Humanoids 2004, Nov; Los Angeles (USA).

Schiele A, van der Helm FCT. 2006. Kinematic design to improve ergonomics in human machine interaction. Proc. Neural Systems and Rehabilitation Engineering. IEEE Tran. 14(4):456469.

Schiele A, Visentin G. 2003. The esa human arm exoskeleton for space robotics telepresence. In: 7th International Symposium on Artificial Intelligence, Robotics and Automation in Space (i-SAIRAS).

Tsagarakis NG, Caldwell DG. 2003. Development and control of a soft-actuated exoskeleton for use in physiotherapy and training. Auton. Robots. 15:21-33.

Tsagarakis N, Caldwell D, Merdano-Cerda G. 1999. A 7dof pneumatic muscle actuator powered exoskeleton. Proc. 8th IEEE Int Workshop on Robot and Human Interaction. IEEE. 327333.

Veneman JF, Ekkelenkamp R, Kruidhof R, van der Helm FCT, Kooij H. van der 2006. A series elastic- and bowdencable-based actuation system for use as torque actuator in exoskeleton-type robots. I. J. Robotic Res. 25(3):261-281.

Wells D, Iversen E, Davis C, Jacobsen S. 1990. An investigation of hydraulic actuator performance trade-offs using a generic model. Paper presented at: IEEE International Conference on Robotics and Automation.

\section{Appendix}

\section{A. The exoskeleton prototype}

A first passive version of the device was developed in order to test the chosen kinematic configuration on the human being. It will also help to evaluate the design of the final system in terms of joints alignment, kinematic configuration, workspace limitation, and adaptability on different users. The system (Figure 12) has the same mobility of the model (Figure 11), and overall it has 15 joints, each one equipped with a position sensor.

The exoskeleton is interfaced with the human body via three contact points: lower back (upper shoulder), shoulder (lower shoulder), upper arm and wrist. The kinematic chain (Figure 11) from the back to the shoulder has six DOF (b01-b06), from the shoulder to the upper arm four DOF ( $\mathrm{s} 01-\mathrm{s} 03, \mathrm{ua} 01)$ and from the upper arm to the wrist five DOF. Due to the requirement of having a mechanical structure as stiff as possible, capable to transfer the forces from the back to the wrist and compensate the lengthchanging during the joint movements, we developed a kinematic structure for the forearm that has three additional DOF (e01, e02, la01) (see Figure 11).

The fixing of the exoskeleton to the user is done via special connections, this taking into consideration also the comfort of wearing the exoskeleton. Both the active and passive systems 


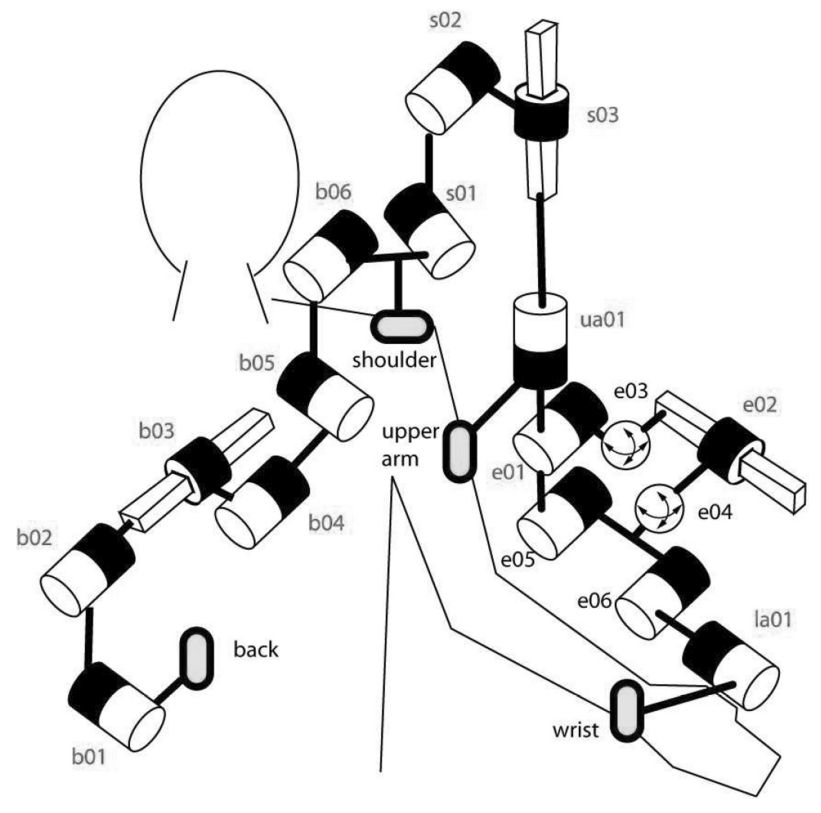

Figure 11. Kinematic chain for the passive exoskeleton.

have to fit as far as possible in order to keep the alignment of the exoskeleton joint to the human joints. For the active exoskeleton it will be even more difficult to distribute the forces from and to the user's limb. For the design of the passive exoskeleton the basic dimensions (requirements) were taken from the male 95 th percentile and female 95 th percentile according to DIN 33402.

The device was tested on three different subjects (Figure 13), two males and one female, with the idea to verify the adaptability of the system to different user sizes, and to validate the chosen kinematic model. Furthermore, this first experience also indicated some improvements to be included and the directions to follow in order to design the mechanics of the active system.

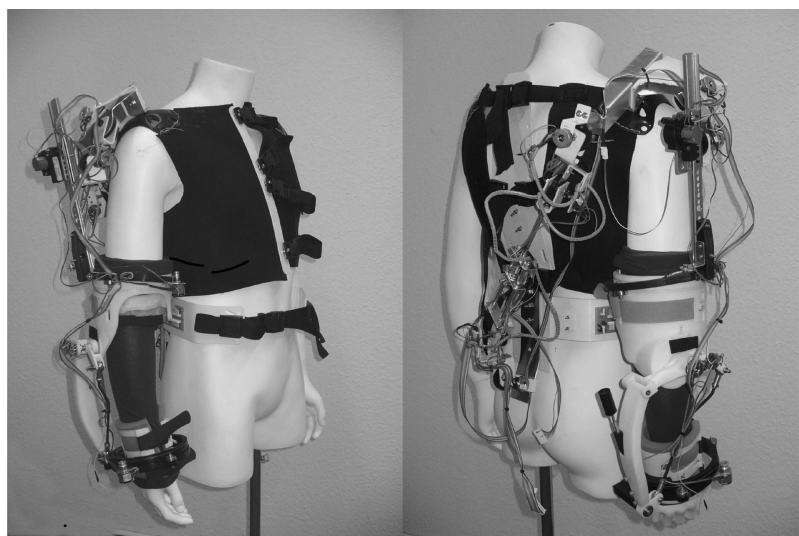

Figure 12. The exoskeleton prototype, lateral and back views.

The exoskeleton actuation system. Because it is not possible to find in the market a suitable hydraulic actuator for low-pressure purposes (until 50 bars, $50 \times 10^{5} \mathrm{~Pa}$ ) and with a compact structure, we decided to develop our own system. The actuator was designed with the intent to keep the device as light as possible. The actual prototype, including the valve system, weights only $0.37 \mathrm{~kg}$ and it is able to deliver a torque up to $60 \mathrm{~N} / \mathrm{m}$ at 50 bars. State of the art electrical DC motors with the same features in terms of torque, speed and response time, normally weigh around $3 \mathrm{~kg}$.

From the technical point of view, the actuation concept (without considering the elastic element) consists of a rotary lowpressure hydraulic actuator for each joint and a miniature hydraulic power unit that can be installed in a separate location. Since this is a custom design, it will be possible to adjust the size, range of motion and output torque according to the requirements for each joint. An optical encoder with 1000 counts per revolution and an index pulse is used to determine the absolute position of the joint and to enable precise speed control. Pressure sensors in both chambers are used to measure the output torque of the actuator in real-time. The hollow shaft in the centre of the actuator can be used to guide hydraulic hoses and wires across the joints.
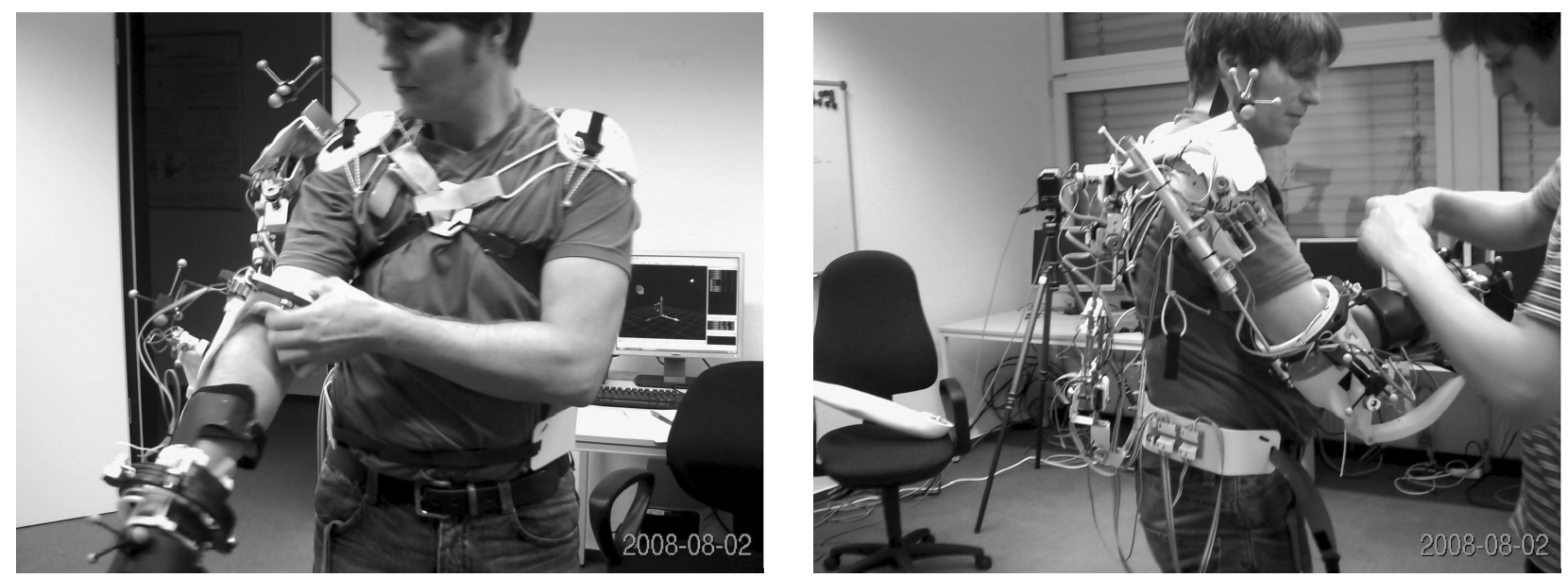

Figure 13. A first user wearing the passive version of the exoskeleton. 


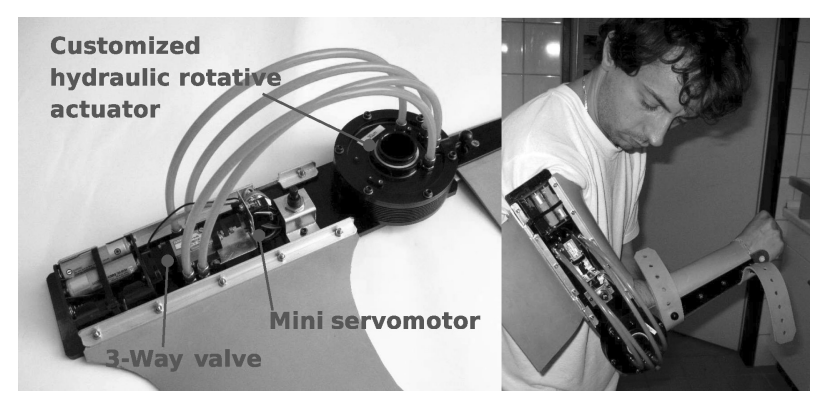

Figure 14. Customised hydraulic rotary actuator (left picture). A user wearing the Elbow haptic interface mounting the actuator and a control system to regulate the damping behaviour of the joint (right picture).

In order to start to test the actuator concept in combination with the haptic interface, a simple 1 DOF elbow-exoskeleton was also developed (Figure 14). The system is equipped with proper fixing belts to ensure the interface on the user's upper and lower arm. In this case it is required also to align the centre of rota- tion of the joint with the elbow's centre of rotation in order to avoid unwanted strains on the limb during the flexion-extension movements. A servo-assisted mini valve is also integrated to regulate the flow and therefore the damping behaviour of the joint. 

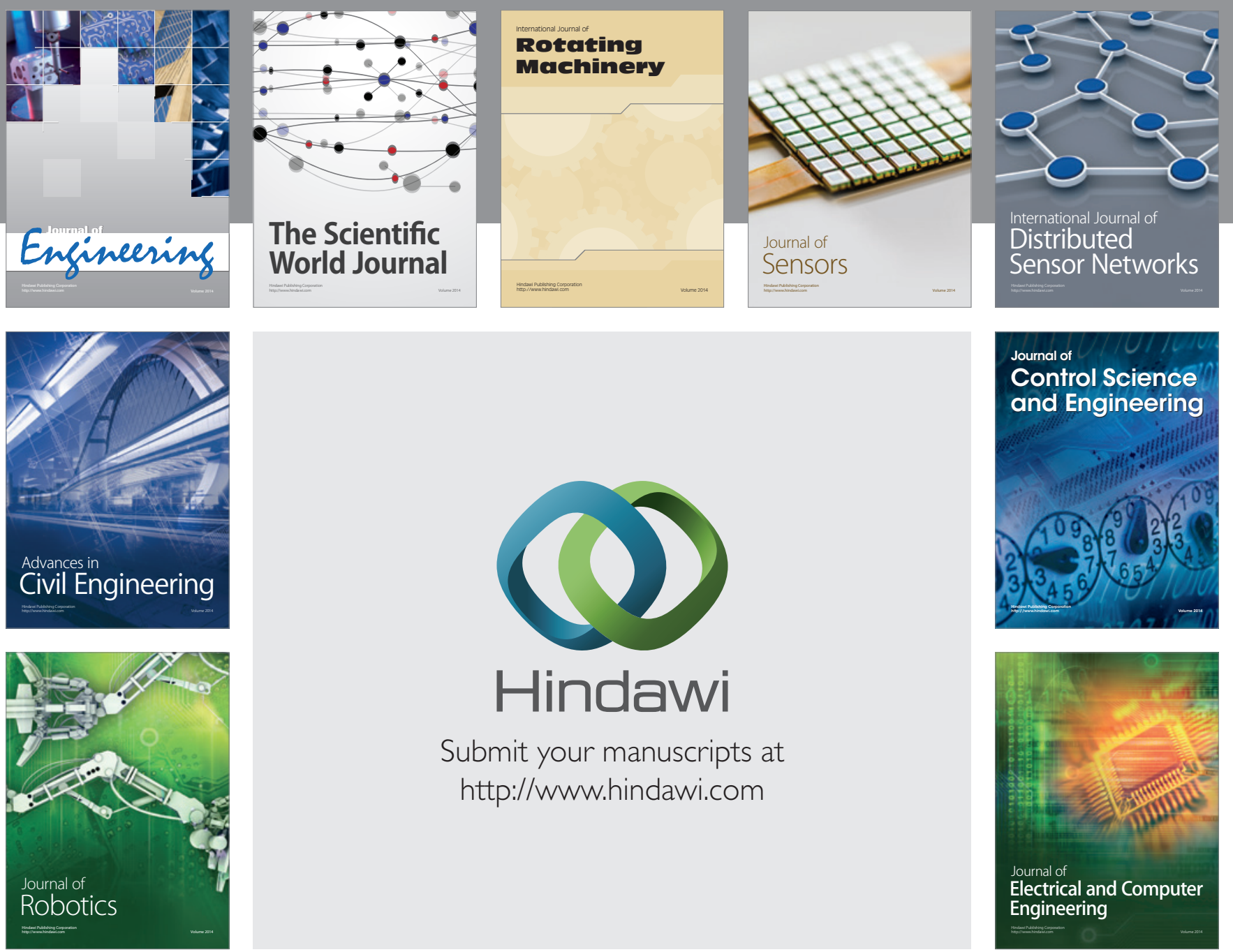

Submit your manuscripts at

http://www.hindawi.com
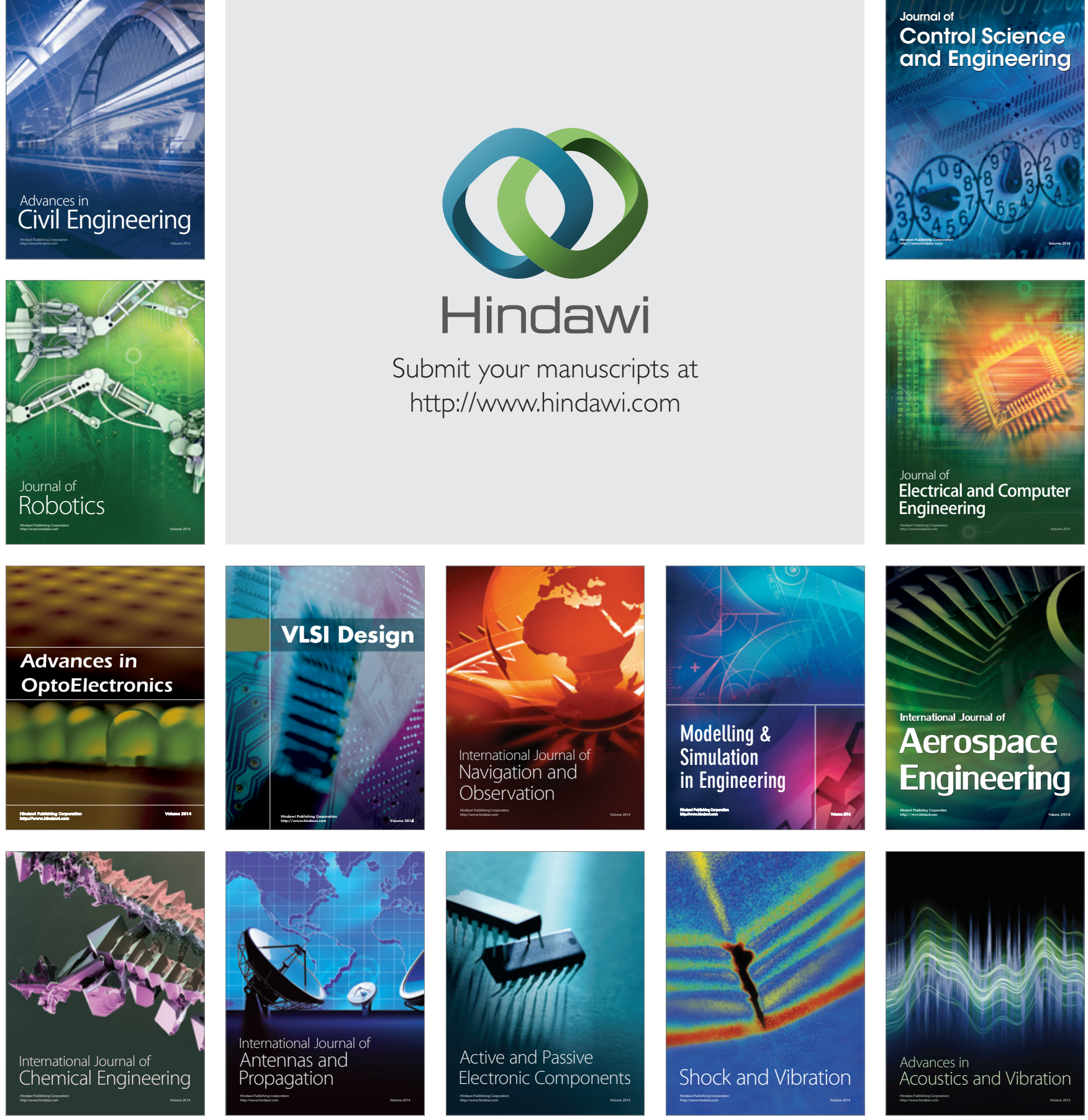